\title{
PERFIL EPIDEMIOLÓGICO DOS NASCIDOS VIVOS EM BELO HORIZONTE, MG, BRASIL
}

\section{EPIDEMIOLOGICAL PROFILE OF LIVE BIRTHS IN BELO HORIZONTE, MG, BRAZIL}

\section{PERFIL EPIDEMIOLÓGICO DE LOS NACIDOS VIVOS EN BELO HORIZONTE, MG, BRASIL}

Verônica Aparecida Ferreira ${ }^{1}$, Nathália Silva Gomes ${ }^{2}$, Cristina Wide Pissetti ${ }^{3}$, Sueli Riul da Silva ${ }^{4}$, Mariana Torreglosa Ruiz ${ }^{5}$.

\section{RESUMO}

Objetivo: este estudo teve por objetivo descrever o perfil epidemiológico dos nascidos vivos residentes no município de Belo Horizonte/MG, no período de 1994 a 2014. Método: trata-se de uma pesquisa transversal, retrospectiva, de abordagem quantitativa, realizada com base nos dados secundários disponíveis no Sistema de Informações sobre Nascidos Vivos. Resultados: os resultados evidenciaram 738.314 nascimentos no município de Belo Horizonte/MG nesse período, sendo a maioria do sexo masculino (51,1\%); a termo (90,2\%); com índice de Apgar entre 08 e 10 no primeiro minuto de vida ( $85 \%$ ); com peso ao nascer entre $2500 \mathrm{~g}$ e $3999 \mathrm{~g}(86,4 \%)$; e sem a presença de anomalias congênitas $(58,2 \%)$. Conclusão: o Sistema de Informações sobre Nascidos Vivos representa um avanço no registro de dados e serve de subsídio para o planejamento e a implantação de políticas públicas voltadas para a saúde materno-infantil.

Descritores: Nascimento vivo; Sistemas de informação em saúde; Saúde da criança.

\section{ABSTRACT}

Objective: this study aimed to describe the epidemiological profile of live births living in the city of Belo Horizonte, MG, from 1994 to 2014. Method: this is a cross-sectional, retrospective, quantitative approach based on the secondary data available in the Live Births Information System. Results: the results showed 738,314 births in the city of Belo Horizonte / MG in this period, being the majority male ones (51.1\%); at term (90.2\%); with an Apgar score between 8 and 10 in the first minute of life (85\%); with birth weight between $2500 \mathrm{~g}$ and $3999 \mathrm{~g}(86.4 \%)$; and without the presence of congenital anomalies $(58.2 \%)$. Conclusion: the Live Births Information System represents an advance in the data registry and works as a subsidy for the planning and implementation of public policies focused on maternal and child health.

Descriptors: Live birth; Health information systems; Child health.

\section{RESUMEN}

Objetivo: este estudio tuvo por objetivo describir el perfil epidemiológico de los nacidos vivos residentes en el municipio de Belo Horizonte/MG, en el período de 1994 a 2014. Método: se trata de una pesquisa transversal, retrospectiva, de abordaje cuantitativa, realizada con base en los datos secundarios disponibles en el Sistema de Informaciones sobre Nacidos Vivos. Resultados: los resultados evidenciaron 738.314 nacimientos en el municipio de Belo Horizonte/MG en ese período, siendo la mayoría del sexo masculino (51,1\%); a término (90,2\%); con índice de Apgar entre 08 y 10 en el primero minuto de vida (85\%); con peso al nacer entre $2500 \mathrm{~g}$ y $3999 \mathrm{~g}(86,4 \%)$; y sin la presencia de anomalías congénitas $(58,2 \%)$. Conclusión: el Sistema de Informaciones sobre Nacidos Vivos representa un avanzo en el registro de datos y sirve de subsidio para el planeamiento y la implantación de políticas públicas volteadas para la salud materno-infantil.

Descriptores: Nacimiento vivo; Sistemas de información en salud; Salud del niño.

${ }^{1}$ Graduada em Enfermagem pela Universidade Federal do Triângulo Mineiro. ${ }^{2}$ Graduada em Enfermagem. Mestre em Enfermagem pela Universidade Federal do Triângulo Mineiro. ${ }^{3} \mathrm{Graduada}$ em Biomedicina pela Universidade Federal da Paraíba. ${ }^{4}$ Graduada em Enfermagem e Obstetrícia. Doutora em Enfermagem Fundamental pela Escola de Enfermagem de Ribeirão Preto/USP. Docente na Universidade Federal do Triângulo Mineiro. ${ }^{5}$ Graduada em Enfermagem. Doutora em Ciências pela Escola de Enfermagem de Ribeirão Preto/USP. Docente na Universidade Federal do Triângulo Mineiro.

Como citar este artigo:

Ferreira VA, Gomes NS, Pissetti CW, et al. Perfil Epidemiológico dos Nascidos Vivos em Belo Horizonte, MG, Brasil. 2018;8:e2550. [Access ; Available in: DOI: http://dx.doi.org/10.19175/recom.v8i0.2550 


\section{INTRODUÇÃO}

No Brasil, desde a década de 70, informações sobre nascimentos eram baseadas, exclusivamente, no Sistema de Registro Civil e divulgadas pelo Instituto Brasileiro de Geografia e Estatística (IBGE) ${ }^{(1)}$. Em decorrência da existência de sub-registro de nascimentos e da importância das informações sobre os nascidos vivos para as estatísticas de saúde, epidemiologia e demografia, o Ministério da Saúde (MS) implantou, em 1990, o Sistema de Informações sobre Nascidos Vivos (SINASC) ${ }^{(2)}$.

O SINASC baseia-se nos dados contidos na Declaração de Nascido Vivo (DNV) - documento oficial e padronizado para todo o país. A DNV é impressa em três vias previamente numeradas, sob a responsabilidade do MS, por meio do Departamento de Análise da Situação de Saúde (DASIS - SVS). O documento é distribuído gratuitamente às Secretarias Estaduais de Saúde, que remetem às Secretarias Municipais de Saúde e estas, por sua vez, repassam aos estabelecimentos de saúde e cartórios ${ }^{(3)}$.

Por intermédio desses registros é possível subsidiar intervenções relacionadas à saúde da mulher e da criança para todos os níveis de atenção do Sistema Único de Saúde (SUS), especialmente, ações de atenção à gestante e ao recém-nascido (RN), pois são registrados nas DNV, dados sobre a mãe e o recém-nascido, compondo o perfil epidemiológico dos nascimentos. Além disto, o acompanhamento da evolução das séries históricas do SINASC permite a identificação de prioridades de intervenção, o que contribui para efetiva melhoria do sistema ${ }^{(3)}$.

Contextualizando, em Belo Horizonte/MG, o processo de implantação do SINASC iniciou-se no segundo semestre de 1991, sendo implantado, definitivamente, a partir de 15 de fevereiro de $1992^{(4)}$. O município de Belo Horizonte é a capital do estado de Minas Gerais e, atualmente, possui uma população estimada em 2.491.109 habitantes ${ }^{(5)}$.

Diante desse contexto, o presente estudo teve como objetivo descrever o perfil epidemiológico dos nascidos vivos residentes no município de Belo Horizonte/MG, no período de 1994 a 2014, a partir dos dados obtidos no SINASC.

\section{MÉTODOS}

Trata-se de um estudo transversal, retrospectivo, de abordagem quantitativa, realizado com base nos dados secundários disponíveis no banco de dados do SINASC. Esta base de dados é gerada pelo Departamento de Informática do SUS (DATASUS) em cooperação com o Centro Nacional de Epidemiologia (CENEPI).

A amostra do estudo foi composta por todos os registros de nascidos vivos de mães residentes no município de Belo Horizonte/MG durante o período de janeiro a dezembro dos anos de 1994 a 2014, totalizando 738.314 nascimentos declarados. Este período foi delimitado para estudo por compreender toda a informação disponível no sistema até o atual momento.

As variáveis consideradas de interesse foram: sexo do recém-nascido; peso ao nascer; Apgar no primeiro e no quinto minuto de vida; presença de anomalias congênitas; idade materna; grau de instrução e estado civil da mãe; tipo de gravidez; duração da gestação; tipo de parto; número de consultas pré-natais e local de nascimento.

A coleta de dados ocorreu entre os meses de novembro de 2016 a janeiro de 2017. Os dados constantes do banco SINASC foram exportados, organizados e tabulados no programa Microsoft Excel', importados e analisados pelo aplicativo Statistical Package for the Social Sciences (versão 23). Para a análise, utilizou-se estatística descritiva e os dados numéricos foram apresentados em tabelas, em valores absolutos e percentuais. Para efeito de apresentação tabular, o cálculo das porcentagens foi aproximado em duas casas decimais.

Por tratar-se de estudo realizado com dados secundários, de acesso público, não foi necessária autorização do Comité de Ética em Pesquisa com Seres Humanos para seu desenvolvimento; contudo, ao longo da análise e discussão dos achados, tomou-se o cuidado de respeitar os princípios éticos da pesquisa que envolve seres humanos, sobretudo no que se refere à beneficência e não maleficência dos estudos, conforme preconiza a Res. 466/2012 do Brasil.

\section{RESULTADOS E DISCUSSÃO}

Os registros do SINASC para o município de Belo Horizonte/MG, no período de janeiro de 1994 a dezembro de 2014, podem ser vistos na Figura 1. 
Figura 1 - Distribuição do número de nascimentos no município de Belo Horizonte/MG, conforme ano de nascimento, a partir dos registros do SINASC, 1994 a 2014.

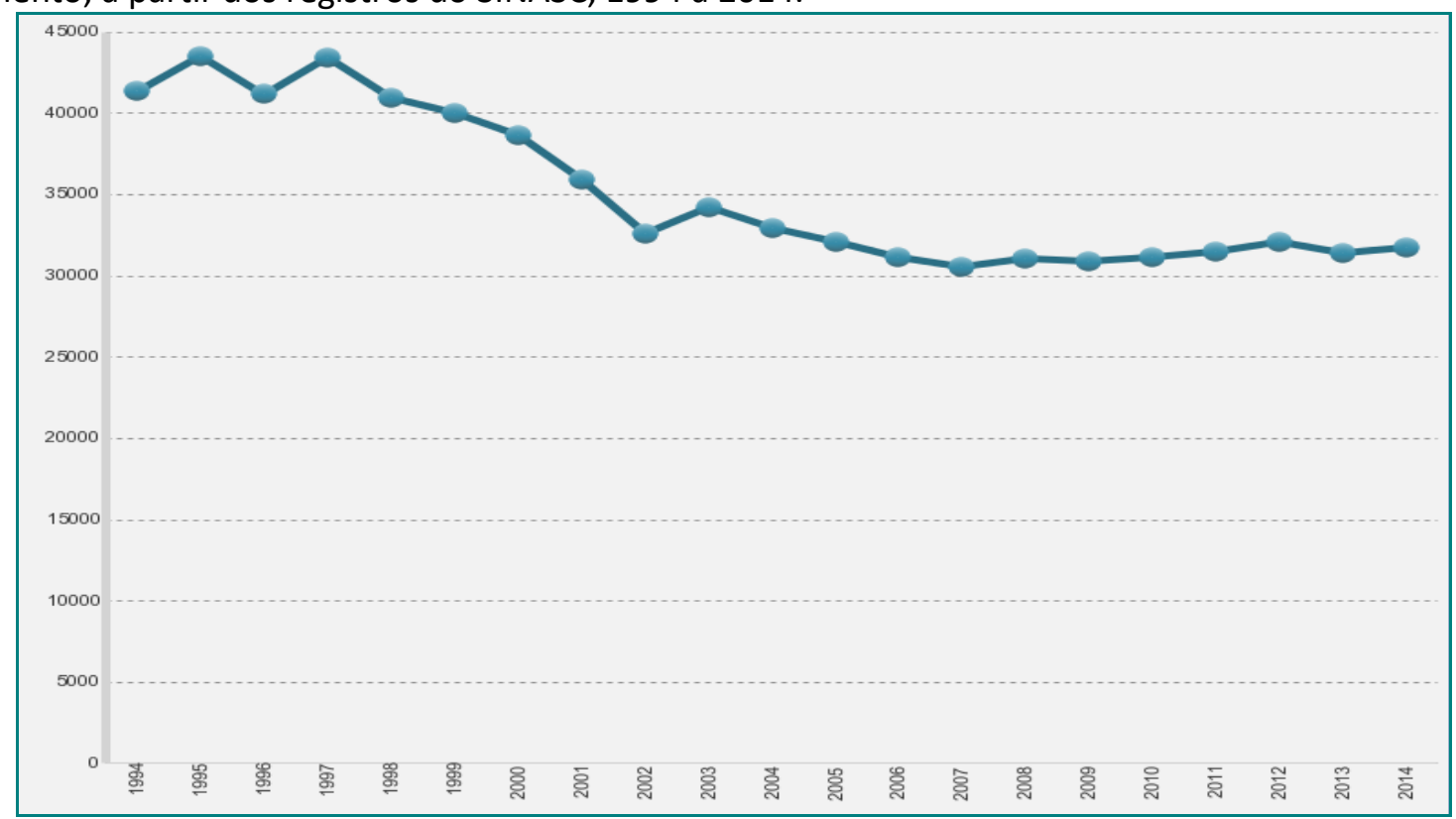

Fonte: Sistema de Informações sobre Nascidos Vivos. Belo Horizonte/MG: 1994-2014.

No período de estudo, foram declarados 738.314 nascimentos no município de Belo Horizonte/ MG; porém, nota-se que a taxa de nascimentos decresceu a partir de 2001, mantendo índices estáveis. Esse fato pode ser justificado, pois, atualmente, a sociedade mundial e brasileira - vive um período denominado de 'transição obstétrica', caracterizado por menores taxas de fertilidade, maior envelhecimento da população obstétrica, predomínio de doenças crônico-degenerativas associado a uma crescente institucionalização do parto ${ }^{(6)}$.
Em relação às características clínicas dos $\mathrm{RN}$, notou-se um discreto predomínio de nascidos vivos do sexo masculino (51,1\%); a maioria $(86,4 \%)$ nasceu pesando entre 2500 e 3999 gramas (considerado peso adequado para $\mathrm{RN}$ a termo); $10,6 \%$ nasceram com baixo peso (inferior a 2500 gramas), $0,7 \%$ desses nasceram com menos de 1000 gramas e $2,9 \%$ eram macrossômicos (peso de nascimento superior a 4000 gramas), conforme dados apresentados na Tabela 1.

Tabela 1 - Caracterização clínica dos nascidos vivos em Belo Horizonte/MG, a partir dos registros do SINASC, 1994 a 2014.

\begin{tabular}{lll}
\hline Sexo & Número & $\%$ \\
Masculino & & 51,1 \\
Feminino & 377.068 & 48,8 \\
Ignorado & 360.658 & 0,1 \\
Peso ao Nascer & 588 & \\
Menor que 1000g & & 0,7 \\
1000g a 2499g & 4.925 & 9,9 \\
2500g a 3999g & 73.088 & 86,4 \\
4000g e mais & 637.807 & 2,9 \\
Ignorado & 21.547 & 0,1 \\
Apgar 10 minuto & 947 & \\
0 a 2 & & 1,4 \\
3 a 5 & 10.083 & 3,6 \\
6 a 7 & 26.974 & 8,6 \\
8 a 10 & 63.197 & 85,0 \\
Ignorado & 627.872 & 1,4 \\
\hline
\end{tabular}


Número

Apgar 5 minuto

\begin{tabular}{lll}
0 a 2 & 3.958 & 0,6 \\
3 a 5 & 4.046 & 0,6 \\
6 a 7 & 13.636 & 1,8 \\
8 a 10 & 704.170 & 95,4 \\
Ignorado & 12.504 & 1,6 \\
Anomalia congênita & & 0,4 \\
Sim & 3.126 & 58,2 \\
Não & 429.432 & 41,4 \\
\hline Ignorado & 305.756 & \\
\hline
\end{tabular}

Fonte: Sistema de Informações sobre Nascidos Vivos. Belo Horizonte/MG: 1994-2014.
$\%$

0,6
0,6
1,8
95,4
1,6

0,4
58,2
41,4

O discreto predomínio de $\mathrm{RN}$ do sexo masculino entre os nascidos vivos foi compatível com dados da literatura. Estudo sobre tendências seculares das proporções de nascimentos entre gêneros na América do Sul, durante a segunda metade do século $\mathrm{XX}$, mostrou que existe uma taxa de nascimentos masculinos que excede a de femininos ${ }^{(7)}$. Ainda, em Belo Horizonte/MG, em uma pesquisa realizada em 1997, que também utilizou a base de dados do SINASC, a proporção encontrada foi de $51 \%{ }^{(4)}$. Entretanto, um estudo, em uma maternidade de risco na região Sul, mostrou discreto predomínio de $\mathrm{RN}$ do sexo feminino, que representou $50,9 \%$ de todos os nascimentos ${ }^{(8)}$.

O percentual de baixo peso ao nascer, obtido nesse estudo, foi de 10,6\%. Esse índice foi superior aos índices encontrados no município da Serra/ES, $8,3 \%^{(9)}$ e também no município de Chapecó/SC, $9,2 \%^{(10)}$. O baixo peso ao nascimento foi uma característica presente em $50,3 \%$ dos nascimentos em uma maternidade de risco $^{(8)} \mathrm{e}$ está associado a casos de morbidade grave ${ }^{(11)} \mathrm{e}$ óbito neonatal ${ }^{(12)}$.

Quanto ao índice de Apgar, percebeu-se que $86,4 \%$ e $95,4 \%$ dos neonatos apresentaram valores entre 08 e 10 no primeiro e quinto minutos de vida, respectivamente. Não houve detecção de anomalia na maioria dos neonatos ao nascimento (58,2\%); entretanto, essa informação foi ignorada ou não preenchida em $41,4 \%$ dos registros. Entre as anomalias detectadas, foram mais frequentes: malformações do aparelho osteomuscular $(30,9 \%)$; outras malformações $(10,8 \%)$; do aparelho genitourinário $(9,5 \%)$, dos pés $(9,0 \%)$ e do sistema nervoso $(8,4 \%)$.

Resultados semelhantes foram encontrados em um estudo realizado entre os anos de 2001 a 2005 no Espírito Santo(9). No estudo conduzido em Chapecó/SC, observou-se que $97,4 \%$ ( $n=5.764)$ dos recém-nascidos alcançaram Apgar $\geq 8$ no 50 minuto de vida ${ }^{(10)} \mathrm{e}$ inferior a dados de uma maternidade de risco do Sul do Brasil, 9,7\% ${ }^{(8)}$. Ressalta-se que índices de Apgar inferiores a sete no quinto minuto de vida do neonato podem indicar morbidade neonatal grave e dificuldade de adaptação extra-uterina ${ }^{(11)}$.

Através do resultado, foi possível constatar que de todas as variáveis, o campo da DNV presença de anomalias apresentou maior número de informações ignoradas ou não preenchidas (41,4\% dos registros), de forma que, dentre as informações disponíveis, $0,4 \%$ dos neonatos possuía algum tipo de anomalia ou malformação, sendo mais frequentes: malformações do aparelho osteomuscular (30,9\%); outras malformações $(10,8 \%)$; do aparelho genitourinário $(9,5 \%)$, dos pés $(9,0 \%)$ e do sistema nervoso $(8,4 \%)$. Um estudo sobre malformações e anomalias encontrou como causas mais comuns malformações do sistema nervoso e do aparelho circulatório $^{(13)}$.

Há que se destacar que as malformações ou anomalias podem causar impacto negativo e transtornos psicológicos para as mães como estresse, ansiedade e depressão ${ }^{(14)}$. Em estudo com mães de filhos com anomalias, identificou-se que a maioria já conhecia o diagnóstico antes do nascimento e essas apresentavam melhores estratégias de adaptação, assim como aquelas cujos filhos estavam internados por períodos prolongados. Mulheres que receberam o diagnóstico no parto ou após o nascimento apresentavam maior tendência ao isolamento. Também verificou-se que $84 \%$ das mães apresentavam estresse psicológico em fase de resistência (sensibilidade emocional excessiva com períodos de intensa irritabilidade) e o restante encontrava-se em período de exaustão, 
ou seja, em processo adaptativo. As mães que estavam, nesse período, receberam o diagnóstico no pré-natal| ${ }^{(14)}$, indicando a importância do diagnóstico precoce para a adaptação da mãe e da família. Além disto, a malformação congênita também é um preditor de morbidade neonatal grave $^{(11)}$.

A apresentação dos dados dos nascidos vivos segundo as características maternas pode ser observada na Tabela 2.

Tabela 2 - Caracterização sociodemográfica das mães de nascidos vivos residentes em Belo Horizonte/MG, a partir dos registros do SINASC, entre os anos de 1994 a 2014.

\begin{tabular}{lll}
\hline Idade da mãe & Número & $\%$ \\
10 a 19 anos & & 14,5 \\
20 a 34 anos & 106.590 & 71,5 \\
35 ou mais & 527.248 & 13,7 \\
Ignorada & 101.175 & 0,3 \\
Estado civil & 2.228 & \\
Solteira & & 32,9 \\
Casada ou união consensual & 242.782 & 31,5 \\
Viúva & 1.449 & 0,2 \\
Separada judicialmente & 8.930 & 1,2 \\
Ignorado & 252.472 & 34,2 \\
Instrução da mãe & & \\
Nenhuma & 5.576 & 0,8 \\
1 a 3 anos & 17.008 & 2,3 \\
4 a 7 anos & 217.073 & 29,4 \\
8 a 11 anos & 295.548 & 40,0 \\
12 anos e mais & 147.845 & 20,0 \\
lgnorado & 55.264 & 7,5 \\
\hline Fonte: Sistema & & \\
\hline
\end{tabular}

Fonte: Sistema de Informações sobre Nascidos Vivos. Belo Horizonte/MG: 1994-2014.

Em relação à idade materna, observou-se que houve predomínio da faixa etária dos 20 aos 34 anos de idade (71,5\%). Pesquisa realizada em Aracaju/SE, no ano de 2010, encontrou proporção de $72,1 \%$ para essa mesma faixa etária ${ }^{(15)}$. Já em estudo em Belo Horizonte/MG, encontrou-se $77 \%$ em 1992 e 75,7\% em 1994 ${ }^{(4)}$. Também, estudo realizado em Maringá/PR, em 2014, encontrou $72,5 \%$ das mães na faixa etária dos 20 aos 34 anos $^{(16)}$, o que faz notar que esse perfil etário tem se mantido no Brasil por pelo menos duas décadas.

Gestantes adolescentes representaram $14,5 \%$ das mães do município de Belo Horizonte/MG no período deste estudo. Esse percentual foi inferior ao observado em outros estudos como em Santa Maria/RS ${ }^{(17)}, 17,2 \%$, em Aracaju/SE ${ }^{(15)}$ com um percentual de $15,5 \%$ e no município de Chapecó/SC $-16,0 \%{ }^{(10)}$.

Observou-se predomínio de mulheres solteiras $(32,9 \%)$ e com parceiro fixo $(31,5 \%)$ no período analisado. Contudo, esse item foi ignorado na maioria das declarações (34,2\%). Estudos sobre perfil apontaram que a maioria das mães não apresentava parceiro fixo ou se declararam solteiras nas seguintes proporções: Aracaju/SE, 70,4\% ${ }^{(15)}$; Chapecó/SC, 18,2\%(10); região Sul do Brasil, $57,2 \%{ }^{(8)}$.

Em relação ao nível de instrução das mães, a maioria (40\%) possuía de 08 a 11 anos de estudos, 20\% apresentavam mais de 12 anos (ensino médio completo ou superior) e $0,8 \%$ era analfabeta. A baixa escolaridade, no entanto, por motivos não justificáveis, apresentou-se como fator de proteção para partos operatórios/cesáreas, quando analisadas as informações da DNV sobre escolaridade materna e tipo de parto ${ }^{(17)}$.

As variáveis relativas à gestação encontram-se na Tabela 3. 
Tabela 3 - Caracterização clínica das gestações dos nascidos vivos residentes em Belo Horizonte/MG, a partir dos registros do SINASC, entre os anos de 1994 a 2014.

\begin{tabular}{|c|c|c|}
\hline & Número & $\%$ \\
\hline \multicolumn{3}{|l|}{ Tipo de gravidez } \\
\hline Única & 720.901 & 97,6 \\
\hline Dupla & 15.822 & 2,1 \\
\hline Tripla e mais & 856 & 0,2 \\
\hline Ignorada & 735 & 0,1 \\
\hline \multicolumn{3}{|l|}{ Tipo de parto } \\
\hline Vaginal & 392.224 & 53,1 \\
\hline Cesáreo & 343.203 & 46,5 \\
\hline Fórceps & 2.005 & 0,3 \\
\hline Ignorado & 882 & 0,1 \\
\hline \multicolumn{3}{|l|}{ Duração da gestação } \\
\hline$<37$ semanas & 62.155 & 8,4 \\
\hline 37 - 41 semanas & 665.691 & 90,2 \\
\hline 42 semanas ou mais & 6.418 & 0,9 \\
\hline Ignorado & 4.050 & 0,5 \\
\hline \multicolumn{3}{|l|}{ Consulta pré-natal } \\
\hline Nenhuma & 9.003 & 1,2 \\
\hline 1 a 3 consultas & 28.115 & 3,8 \\
\hline 4 a 6 consultas & 175.647 & 23,8 \\
\hline 7 ou mais consultas & 416.916 & 56,5 \\
\hline Ignorado & 108.633 & 14,7 \\
\hline \multicolumn{3}{|l|}{ Local do Parto } \\
\hline Hospital & 735.417 & 99,6 \\
\hline Outro estabelecimento & 418 & 0,05 \\
\hline Domicílio & 1.462 & 0,2 \\
\hline Outro & 414 & 0,05 \\
\hline Ignorado & 603 & 0,1 \\
\hline
\end{tabular}

Fonte: Sistema de Informações sobre Nascidos Vivos. Belo Horizonte/MG: 1994-2014.

Observou-se que a gestação foi única em $97,6 \%$ dos casos. Resultado semelhante foi encontrado na pesquisa Nascer no Brasil, um estudo nacional de base hospitalar, o qual verificou que $98 \%$ das gestações foi única e que a gestação múltipla pode ter como consequência o nascimento prematuro, aumentando a taxa de mortalidade neonatal ${ }^{(12)}$.

Apesar do predomínio do parto vaginal (53,1\%), houve um alto índice de parto cesáreo, representando $46,5 \%$ de todas as vias de parto. Verificou-se um alto índice de parto cesáreo $(48,4 \%)$ realizado em Belo Horizonte/MG entre os anos de 2002 e 2011, ultrapassando o recomendado pela Organização Mundial de Saúde que atualmente é de $15 \%{ }^{(18)}$.

A proporção do número de cesáreas tem crescido significativamente nos últimos anos no Brasil, passando de $37,8 \%$ em 2000 para $43,2 \%$ em 2005 e 52,2\% em 2010 ${ }^{(19)}$. Em Viçosa, cidade do estado de Minas Gerais, a proporção de partos cirúrgicos aumentou de 56,8\% em 2001 para $63,3 \%$ em $2007^{(20)}$.

Entretanto, a Organização Mundial da Saúde, na declaração sobre taxas de cesáreas, publicada em 2015, proveniente de um estudo ecológico baseado nas melhores evidências científicas, sugere que esses índices não ultrapassem $10 \%^{(18)}$. Concluiu-se que a cesárea é uma intervenção efetiva para salvar vidas, quando indicada por motivos justificáveis; entretanto, taxas de cesáreas superiores a $10 \%$ não foram associadas à redução de mortalidade e aumentaram os índices de complicações ${ }^{(18)}$. Essa declaração sugere, ainda, que as instituições de saúde que assistem partos adotem a Classificação de Robson para identificar quem são as mulheres que deverão ser submetidas à cesárea e seus possíveis resultados ${ }^{(21)}$.

Os nascimentos a termo ( 37 a 41 semanas de gestação) representaram $90,2 \%$ do total dos nascidos vivos; porém, chama atenção o elevado número de nascimentos de prematuros $(8,4 \%)$. Estudo realizado em Belo Horizonte/MG demonstra que a proporção de pré-termos no ano de 1992 era $5,2 \%$ aumentando para 5,8\% em $1994^{(4)}$.

A prematuridade é um problema de Saúde Pública e estima-se que 15 milhões de crianças nascem prematuramente/ano, ou seja, a cada dez nascimentos, um é prematuro; um milhão de recém-nascidos morrem em decorrência da prematuridade; além de ser uma das principais 
causas de mortalidade por pneumonia em menores de cinco anos de idade. Mundialmente, a taxa de prematuridade varia de 5 a $18 \%$, sendo que temos de destacar que o Brasil ocupa o $10^{\circ}$ lugar no ranking dos países com maior número de nascimentos prematuros (279 mil) - 11,7\%(22).

Observa-se aumento no número de nascidos vivos pré-termo, concomitantemente ao aumento do número de partos cesáreos, o que nos remete à reflexão de que, possivelmente, há influência da via de parto sobre os nascimentos prematuros. Autores apontam ainda que um número considerável de prematuros nasce por cesariana, principalmente, em casos de gravidez com idade materna avançada (acima dos 35 anos de idade) $)^{(23)}$.

Em relação ao pré-natal, $56,5 \%$ das mães estudadas realizaram sete ou mais consultas e $1,2 \%$ não realizou nenhuma consulta. Esse achado vai de encontro ao estudo realizado em Serra/ES que revelou que a maioria $(53,3 \%)$ das mulheres tinha feito sete ou mais consultas de pré-natal e $1,9 \%$ não tinha realizado nenhuma ${ }^{(9)}$. Já um estudo realizado em João Pessoa/PB apontou que $90 \%$ das gestantes fizeram mais de seis consultas pré-natal(24).

Cabe destacar que o objetivo da assistência pré-natal é acolher a mulher desde o início de sua gravidez. O controle pré-natal deve ter início precoce, ter cobertura universal, ser realizado de forma periódica e estar integrado com as demais ações preventivas e curativas. Além disso, para ser considerado adequado, deve ser realizado um número mínimo de seis consultas ${ }^{(24)}$.

Houve predomínio dos nascimentos no ambiente hospitalar, representando $99,6 \%$ dos casos pesquisados em Belo Horizonte/MG. Os partos no domicílio ocupam a segunda posição com $0,2 \%$ dos partos. Tem-se observado um movimento de estímulo e incentivo ao parto domiciliar com a presença de familiares e sendo assistido por profissionais médicos e enfermeiros capacitados. Esta ocorrência é relativamente rara e praticada, principalmente, por mulheres com condições socioeconômicas e culturais elevadas.

A pesquisa Nascer no Brasil, que traçou o perfil dos nascimentos brasileiros, verificou associação do baixo peso ao nascimento, gestações de alto risco e condições de adaptação do RN com o aumento do número de óbitos neonatais. De acordo ainda com este estudo, a peregrinação por um leito de terapia intensiva (CTI neonatal) ainda é uma lacuna assistencial das gestações de alto risco e óbitos por asfixia e prematuridade refletem a falta de qualidade assistencial pois, nesses casos, a chance de evitabilidade é grande e associa-se com a assistência pré-natal e ao parto ${ }^{(12)}$.

Há que se destacar o grande número de informações ignoradas em todas as variáveis investigadas, especialmente: presença de anomalia (41,4\%); estado civil materno $(34,2 \%)$, número de consultas pré-natal $(14,7 \%)$; grau de instrução da mãe $(7,5 \%)$, índice de Apgar no quinto minuto $(1,6 \%)$ e no primeiro minuto $(1,4 \%)$, e duração da gestação $(0,5 \%)$.

De acordo com classificação vigente, podese dizer que as variáveis das DNV tiveram preenchimento excelente quando a variável apresentou menos de $5 \%$ de preenchimento incompleto; bom $(5,0$ a $9,9 \%)$; regular $(10,0$ a $19,9 \%) ; \operatorname{ruim}(20,0$ a $49,9 \%) ;$ e muito ruim $(50,0 \%$ ou mais) ${ }^{(25)}$. Assim, as variáveis anomalia e estado civil tiveram preenchimento ruim; número de consultas pré-natal foi considerado regular e as demais informações foram consideradas boas ou excelentes. Entretanto, assim como em outro estudo que avaliou as variáveis e classificou seu preenchimento como bom ou excelente ${ }^{(25)}$, os autores ressaltam que, por se tratar de dado secundário, não é possível avaliar a confiabilidade dos mesmos.

Ante o exposto, citamos que este estudo teve, como limitação, o uso de informações secundárias produzidas pelo SINASC e, como foi dito anteriormente, não há como se assegurar a confiabilidade dos dados apresentados. Ressaltase a necessidade de enfatizar a importância do preenchimento correto das DNV, fornecendo informações precisas e fidedignas, a fim de se repensar as políticas e estratégias de saúde para o nascimento de RN saudáveis.

\section{CONCLUSÕES}

A partir dos resultados obtidos no município de Belo Horizonte/MG, entre os anos de 1994 a 2014, o perfil de nascidos vivos foi: a quase totalidade dos partos ocorreu em hospitais e com gravidez única; houve predomínio de recém-nascidos do sexo masculino, a termo, com índices de Apgar entre oito e dez no primeiro e quinto minutos de vida, peso ao nascer entre $2500 \mathrm{~g}$ e $3999 \mathrm{~g}$ e sem a presença de anomalias congênitas; a maioria das mães possuía idade entre 20 e 34 anos, era solteira e tinha de oito a 11 anos de estudo; verificou-se um percentual elevado de partos cesáreos e de realização de sete ou mais consultas pré-natais. 
Observa-se, ainda, um número considerável de RN com Apgar inferior a oito no primeiro minuto de vida; de informações ignoradas no item 'anomalia congênita'; de gestação na adolescência; mães com pouca ou nenhuma instrução; nascidos vivos pré-termo e de realização de menos de seis consultas prénatais. Demonstra-se, assim, a necessidade de uma assistência integral à saúde da mulher e do neonato, que a envolva desde o planejamento da gestação até os primeiros anos de vida da criança.

O SINASC possibilita a caracterização do perfil dos nascidos vivos através da disponibilização de informações fundamentais encontradas nas DNV. Apesar das dificuldades relacionadas à alimentação do banco de dados e preenchimento adequado da DNV, o SINASC representa um avanço no registro de dados e serve de subsídio para o planejamento e a implantação de políticas públicas voltadas para a saúde materno-infantil.

\section{REFERÊNCIAS}

1. Pereira CCB, Vidal AS, Carvalho PI, Frias PG. Avaliação da implantação do Sistema de Informações sobre Nascidos Vivos (SINASC) em Pernambuco. Rev Bras Saúde Mater Infant. 2013;13(1):39-49. DOI: 10.1590/S151938292013000100005

2. Melo EC, Mathias TAF. Características das mães e dos recém-nascidos em municípios-sede de regional de saúde no Paraná a partir dos dados do SINASC. Cogitare Enferm. 2010;15(2):293-301. DOI: $10.5380 / c e . v 15 i 2.17864$

3. Ministério da Saúde. Sistema de Informações sobre Nascidos Vivos - SINASC. Brasília: Ministério da Saúde; 2017.

4. Rodrigues $C S$, Magalhães Júnior $H M$, Evangelista PA, Ladeira RM, Laudares S. Perfil dos nascidos vivos no Município de Belo Horizonte, 1992-1994. Cad Saúde Pública 1997 15];13(1):537. DOI: $10.1590 / 50102-311 \times 1997000100014$

5. Instituto Brasileiro de Geografia e Estatística [Internet]. Estimativa da População de Belo Horizonte em 2014. Belo Horizonte: IBGE; 2014.

6. Souza JP, Pillegi-Castro C. Sobre o parto e o nascer: a importância da prevenção quaternária. Cad Saúde Pública. 2014[citado 2017 Jan 15]; 30(sup 11):11-3. DOI: $\underline{10.1590 / 0102-}$ 311XPE02S114

7. Grech V. Secular trends in sex ratios at birth in South America over the second half of the 20th century. J Pediatr. 2013;89(5):505-9. DOI: 10.1016/j.jped.2013.01.006
8. Melo WA, Uchimura TT. Perfil e processo da assistência prestada ao recém-nascido de risco no sul do Brasil. Rev Bras Epidemiol. 2011;14 (2):32337. DOU: $10.1590 /$ S1415-790X2011000200013

9. Lima EFA, Sousa AI, Melo ECP, Caniçali Primo C, Leite FMC. Perfil de nascimentos de um município: um estudo de coorte. Rev Bras Pesqui Saúde 2012;14(1):12-8. DOI: 10. 21722/rbps.v0i0.3404

10. Silva RA, Ferraz L, Busato MA. Perfil epidemiológico dos nascidos vivos no município de Chapecó-SC. RECIIS 2016;10(2):1-16. DOI: 10.29397/reciis.v10i2.1037

11. Silva AAM, Leite AJM, Lamy ZC, Moreira MEL, Gurgel RQ, Cunha AJLA, et al. Morbidade neonatal near miss na pesquisa Nascer no Brasil. Cad Saúde Pública 2014;30(supl 1):182-91. DOI: 10.1590/0102-311X00129613

12. Lansky S, Friche AAL, Silva AAM, Campos D, Bittencourt SDA, Carvalho MC, et al. Pesquisa Nascer no Brasil: perfil da mortalidade neonatal e avaliação da assistência à gestante e ao recémnascido. Cad Saúde Pública 2014;30(supl 1):192207. DOI: $10.1590 / 0102-311 \times 00133213$

13. Gili JA, Poletta FA, Pawluk M, Gimenez LG, Campaña $H$, Castilla $E$, et al. High birth prevalence rates for congenital anomalies in South American regions. Epidemiology 2015;26(5):e53-e55. DOI: 10.1097/EDE.0000000000000345

14. Vicente SRCRM, Paula KMP, Silva FF, Mancini $\mathrm{CN}$, Muniz SA. Estresse, ansiedade, depressão e coping materno na anomalia congênita. Estud Psicol. 2016;21(2):104-16. DOI: 10.5935/16784669.20160011

15. Cravo EO, Oliveira JVR. Perfil epidemiológico dos nascidos vivos no município de Aracaju Sergipe, Brasil. Idéias Inovação 2012 [citado em 15 jan 2017]; 1(1):9-17. Available in: https://periodicos.set.edu.br/index.php/ideiasein ovacao/article/view/282/158

16. Novaes ES, Oliveira RR, Melo EC, Varela PLR, Mathias TAF. Perfil obstétrico de usuárias do Sistema Único de Saúde após implantação da Rede Mãe Paranaense. Ciênc Cuid Saúde 2015 [citado em 29 abr 2018]; 14(4):1436-44. Available in:

http://periodicos.uem.br/ojs/index.php/CiencCui dSaude/article/view/27343/16487

17. Padilha JF, Farinha LB, Mattos KM, Gasparetto

A. Caracterização da saúde materna em Santa Maria, RS. 2005-2009. Rev Enferm UFSM 2012;2(1):79-87. DOI: 10.5902/217976924082 18. Organização Mundial de Saúde. Declaração da OMS sobre Taxas de Cesáreas. Genebra: OMS; 
2015 [citado em 15 jan 2017]. Available in: http://apps.who.int/iris/bitstream/10665/161442/3 WWO RHR 15.02 por.pdf?ua=1\&ua=1

19. Chiavegatto Filho ADP. Partos cesáreos e a escolha da data de nascimento no Município de São Paulo. Ciênc Saúde Coletiva 2013;18 (8):241320. DOI: $10.1590 / \mathrm{S} 1413-81232013000800026$

20. Pereira Júnior AC, Henriques BD. Perfil dos nascidos vivos em Viçosa, Minas Gerais, no período de 2001 a 2007. Rev Méd Minas Gerais 2010 [citado em 15 jan 2017]; 20(4):508-13. Available in: file:///C:/Users/Home/Downloads/v20n4a07.pdf 21. Betran AP, Torloni MR, Zhang J, Ye J, Mikolajczyk R, Deneux-Tharaux $C$, et al. What is the optimal rate of caesarean section at population level? A systematic review of ecologic studies. Reprod Health 2015;12(1):57. DOI: 10.1186/s12978-015-0043-6

22. Blencowe $H$, Cousens $S$, Oestergaard $M$, Chou $D$, Moller $A B$, Narwal $R$, et al. National, regional and worldwide estimates of preterm birth. Lancet 2012;9(379):2162-72. DOI: 10.1016/S01406736(12)60820-4

23. Caetano LS, Luciana Netto, Manduca JNL. Gravidez depois dos 35 anos: uma revisão sistemática da literatura. Rev Min Enferm. 2011;15(4):579-87. DOI: S141527622011000400015

24. Silva EP, Lima RT, Ferreira NLS, Costa MJC e. Pré-natal na atenção primária do município de João Pessoa-PB: caracterização de serviços e usuários. Rev Bras Saúde Mater Infant. 2013;13(1):29-37. DOI: $\quad$ 10.1590/S151938292013000100004

25. Silva RS, Oliveira CM, Ferreira DKS, Bonfim CV. Avaliação da completitude das variáveis do Sistema de Informações sobre Nascidos Vivos SINASC - nos Estados da região Nordeste do Brasil, 2000 e 2009. Epidemiol Serv Saúde 2013; 22(2):347-52. DOI: 10.5123/S1679$\underline{49742013000200016}$

Nota: o presente estudo resultou de monografia de final de curso de graduação em Enfermagem e não recebeu fomento para seu desenvolvimento.

Recebido em: 10/10/2017

Aprovado em: 16/07/2018

Endereço de correspondência:

Sueli Riul da Silva

Rua Donaldo Silvestre Cicci, 665.

CEP: 38082-166 - Uberaba/MG - Brasil

E-mail: sueliriul@terra.com.br 